\title{
Isquemia mesentérica aguda secundaria a síndrome de anticuerpos antifosfolípidos catastrófico. Informe de caso
}

\author{
Acute mesenteric ischaemia secondary to catastrophic \\ antiphospholipid syndrome (CAPS): A case report
}

\author{
Eduardo Cordero Estrada, * Cesar Ploneda Valencia, * Gerardo Castañeda González,* \\ Luis A Ibarra Verdugo, ${ }^{*}$ Carlos R López Lizárraga, ${ }^{*}$ Mónica Abud González*
}

\footnotetext{
Palabras clave:

Isquemia mesentérica,

síndrome de

anticuerpos

antifosfolípidos.
}

Key words:

Mesenteric ischemia, antiphospholipid antibody syndrome.
* Organismo público descentralizado de hospitales civiles de Guadalajara, Hospital Civil Dr. Juan I.

Menchaca, Guadalajara, Jalisco, México.

Recibido: 01/01/2015 Aceptado: 01/05/2015

\section{RESUMEN}

Introducción: El síndrome de anticuerpos antifosfolípidos catastrófico (SAFC) se caracteriza por trombosis intravascular diseminada con isquemia multiorgánica, éste es una variante del síndrome de anticuerpos antifosfolípidos (SAF), la prevalencia de esta enfermedad aún no se tiene registrada por los pocos casos documentados. Informe de caso: Femenina de 16 años de edad, con antecedente de ingesta de cocaína, acude por dolor abdominal de 12 horas de evolución, súbito; náusea y vómitos, dificultad respiratoria, hematoquecia no cuantificada, Glasgow 8; con tensión arterial media de 50 , frecuencia cardiaca de 120 , se pasa a laparotomía exploradora encontrando la totalidad del colon hasta la unión recto-sigmoidea y $30 \mathrm{~cm}$ de íleon distal isquémico sin evidencia de perforación intestinal. Se realiza resección del segmento isquémico con ileostomía terminal, cierre en Hartmann y colocación de bolsa de Bogotá para realizar laparotomía de reevaluación. Se le cataloga con SAF por presentar anticuerpos antinucleares, anticuerpos anticardiolipina y B2 glucoproteína positivos, además de falla renal, deterioro del estado neurológico, falla hepática aguda, trombosis en la pieza resecada y anticuerpos antifosfolípidos positivos, cumpliendo con los criterios para el diagnóstico de SAFC. Discusión: Se cuenta con muy poca información acerca de la asociación de la isquemia mesentérica como hallazgo de presentación del SAFC, en revisiones sistemáticas en PubMed y Medline, no se encontraron artículos que hablaran de la asociación del SAFC y el consumo de cocaína; además, no existen datos del porcentaje de pacientes con SAFC que comiencen con isquemia mesentérica aguda, en diagnósticos donde al cirujano le queda la duda de si la isquemia continuará o no conoce la causa primaria de la misma, se debería realizar un "second look", por la posibilidad de neoisquemia y una nueva resección intestinal. Conclusión: La isquemia mesentérica aguda secundaria al SAFC

\section{ABSTRACT}

Introduction: The catastrophic antiphospholipid syndrome (CAPS) is characterized by disseminated intravascular thrombosis with multiple organ ischemia, this is a variant of antiphospholipid antibodies syndrome (APAS), the prevalence of this disease has not yet been registered because of the scarse documented cases. Case report: Women 16 years old with a history of cocaine use, come up for abdominal pain 12 hours after onset, sudden; nausea and vomiting, difficulty breathing, unquantified hematochezia, Glasgow 8; with mean blood pressure of 50, heart rate of 120 , is passed to exploratory laparotomy finding the entire colon to the rectosigmoid junction and $30 \mathrm{~cm}$ distal ileum ischemic without evidence of intestinal perforation. Ischemic segment resection is performed with terminal ileostomy, Hartmann closure and placement of Bogota bag for laparotomy reassessment. He is cataloged with APAS for presenting antinuclear antibodies, anticardiolipin antibodies and B2 positive glycoprotein, as well as kidney failure, deterioration of neurological status, acute liver failure, thrombosis in the resected specimen and positive antiphospholipid antibodies, meeting the criteria for the diagnosis of CAPS. Discussion: There is very little information about the association of mesenteric ischemia finding SAFC presentation in systematic reviews in PubMed and Medline, no articles were about the association of SAFC and cocaine were found; moreover, there are no data on the percentage of patients with SAFC beginning with acute mesenteric ischemia, where the surgeon diagnoses doubt whether ischemia continue or does not know the primary cause of it, there must be a "second look", for the possibility of a new neoisquemia and bowel resection. Conclusion: The acute mesenteric ischemia secondary to SAFC represents an extremely rare condition with high mortality and therefore, requires a timely surgical treatment and high diagnostic 
representa una entidad sumamente rara con mortalidad elevada y, por lo tanto, se requiere de un tratamiento quirúrgico oportuno y una alta presunción diagnóstica para mejorar la supervivencia; siempre se deberá de tener en cuenta en pacientes jóvenes con pocos o nulos factores de riesgo predisponentes. presumption to improve survival; you should always keep in mind in young patients with scarse or no predisposing risk factors.

\section{INTRODUCCIÓN}

$\mathrm{E}$ I síndrome de anticuerpos antifosfolípidos (SAF) es una entidad autoinmune caracterizada por trombosis venosa, arterial y/o de vasos pequeños de manera recurrente asociada con pérdida de embarazos; ${ }^{1-7}$ existe una variante en la presentación del cuadro denominada síndrome de anticuerpos antifosfolípidos catastrófico (SAFC), la cual se caracteriza por trombosis intravascular diseminada con isquemia multiorgánica, pero la prevalencia no se tiene registrada por los pocos casos conocidos. ${ }^{1,2}$

La prevalencia de la isquemia mesentérica es aproximadamente del $0.1 \%$ a nivel mundial. De acuerdo con Cervera y colaboradores, ${ }^{4}$ los factores desencadenantes como infección, neoplasia, trauma y cirugía se presentan apro-

Cuadro I. Criterios diagnósticos revisados de Sapporo o criterios de Sidney para el diagnóstico del síndrome de anticuerpos antifosfolípidos.

\section{Clínicos}

Trombosis vascular, con estudio de imagen diagnóstico o evidencia histopatológica en cualquier tejido u órgano.

Pérdida fetal sin otra explicación $>10$ SDG de un feto morfológicamente normal.

Uno o más embarazos prematuros $<34$ SDG.

Tres o más pérdidas fetales $<10$ SDG sin otra explicación.

aAP = Anticuerpos antifosfolípidos. $\mathrm{AB} 2 \mathrm{GP}=$ Anticuerpos antibeta 2 glucoproteína .

SDG = Semanas de gestación .

\section{Laboratorio}

Presencia de aAP en dos o más ocasiones, con al menos 12 semanas de diferencia y menos de cinco años de haber iniciado las manifestaciones clínicas.

Uno o más de los siguientes:

$\operatorname{IgG} y / 0 \operatorname{IgM}>40 \mathrm{UI}$ IgG o IgM de AB2GP en títulos > 99\% del percentil del laboratorio. ximadamente en $5 \%$ de los pacientes con SAF $y$ hasta en $30 \%$ con SAFC.

Para el diagnóstico del SAFC se requiere de criterios de laboratorio y clínicos (Cuadros I y II), siendo la isquemia mesentérica una causa rara de presentación; hasta en 50\% de los individuos con SAF la manifestación inicial es el dolor abdominal. ${ }^{1-6}$

El tratamiento del SAFC requiere de tratamiento quirúrgico en hasta el $70 \%$ y múltiples intervenciones en el 20\%. El tratamiento médico agudo es a través de anticoagulantes, glucocorticoides (86\%), plasmaféresis (39\%), ciclofosfamida (36\%), inmunoglobulinas intravenosas $(22 \%)$ y antiagregantes plaquetarios (10\%), según la severidad del cuadro, con una mortalidad al $30 \%$ con este tratamiento y probabilidad de un nuevo episodio de trombosis de cualquier órgano del 10\% en un año. ${ }^{8,9}$

Se consideró trascendente la publicación de este caso, ya que en la búsqueda en los últimos 10 años en PubMed, Medline y Science Citation Index hasta este momento no se encuentra reportada la asociación entre la ingesta de cocaína, el SAFC y la isquemia mesentérica aguda, y de acuerdo con Cervera y su grupo, ${ }^{4}$ hasta este momento existen sólo 114 casos publicados en los que se asocian el SAF y el SAFC con la isquemia mesentérica aguda. ${ }^{4}$

\section{INFORME DE CASO}

Paciente femenina de 16 años de edad, estudiante de preparatoria; como único antecedente patológico de importancia refiere la ingesta de cocaína una vez/semana durante los últimos dos meses. Acude al Hospital Civil Dr. Juan I. Menchaca por presentar dolor abdominal generalizado de 12 horas de evolución, de aparición súbita; náusea y vómitos incontables, dificultad respiratoria, hematoquecia no cuantificada, 
Cuadro II. Criterios diagnósticos del síndrome de anticuerpos antifosfolípidos catastrófico.

1. Tres o más órganos/tejidos involucrados.

2. Manifestaciones clínicas en menos de una semana.

3. Evidencia histológica de trombosis intravascular.

4. Presencia de aAP en dos 0 más ocasiones al menos con seis semanas de diferencia.
Diagnóstico definitivo:

Cumple los cuatro criterios.

Diagnóstico probable:

1,2 y 4 .

1,3 y 4 ; un evento ocurre en el primer mes a pesar de la anticoagulación.

1-4 con sólo dos órganos/tejidos involucrados.

1-4 sin poder corroborar los aAP, ya que la paciente falleció.

aAP = Anticuerpos antifosfolípidos. SAFC = Síndrome de anticuerpos antifosfolípidos catastrófico.

Glasgow 8; con tensión arterial media de 50, frecuencia cardiaca de 120 . Se decide intubación endotraqueal y es revisada por el Servicio de Cirugía General, el cual decide pasar a LAPE (laparotomía exploradora) con diagnóstico de abdomen agudo, probable embarazo ectópico roto versus perforación intestinal. Durante el acto quirúrgico se encuentra la totalidad del colon hasta la unión recto-sigmoidea y $30 \mathrm{~cm}$ de íleon distal isquémico (Figuras 1 y 2), sin evidencia de perforación intestinal. Se realiza resección del segmento isquémico con ileostomía terminal, cierre en Hartmann y colocación de bolsa de Bogotá para realizar laparotomía de reevaluación (second look) (Figura 3). Por las condiciones de la paciente, pasa a la Unidad de Terapia Intensiva, donde se le cataloga como SAF (Cuadro I) por presentar anticuerpos antinucleares, anticuerpos anticardiolipina y B2 glucoproteína positivos, además de falla renal, deterioro del estado neurológico, falla hepática aguda, trombosis en la pieza resecada y anticuerpos antifosfolípidos positivos, cumpliendo con los criterios para el diagnóstico de SAFC (Cuadro II).

Estancia intrahospitalaria de 40 días en la Unidad de Terapia Intensiva, fase 3 ventilatoria durante 25 días por presentar neumonía asociada con ventilador y 20 días en planta de cirugía general, realizándose cinco lavados abdominales y cierre de pared con técnica de Gibson (Figura 4) en el último acto quirúrgico.

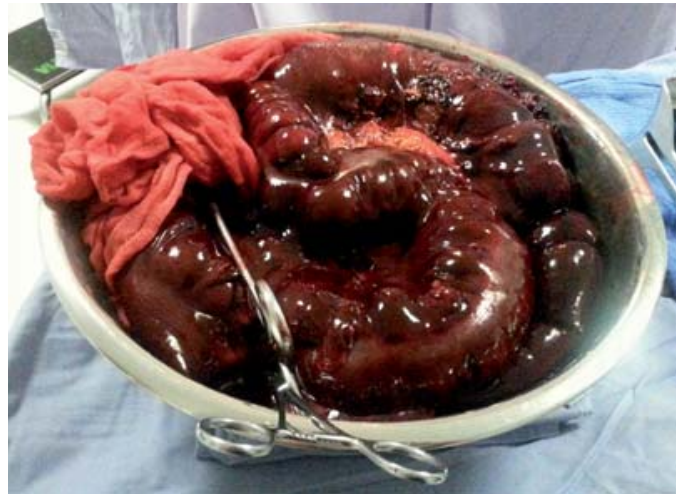

Figura 1. Colon e íleon terminal resecado.

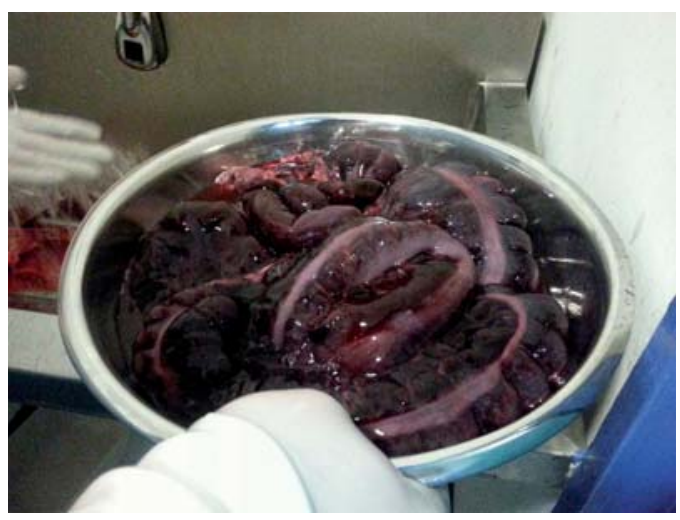

Figura 2. Colon e íleon terminal resecado.

Es tratada con enoxaparina a $40 \mathrm{mg} / 12 \mathrm{~h}$, nutrición parenteral total y antibioticoterapia, con adecuada respuesta clínica. La paciente es dada de alta por su adecuada evolución, siendo tratada actualmente por el Servicio de Reumatología mediante warfarina y dexametasona; se le piden anticuerpos antinucleares tres meses después de su ingreso hospitalario, los cuales resultan positivos, y se confirma el diagnóstico de SAFC.

\section{DISCUSIÓN}

Creemos que este caso clínico es de relevancia, ya que muestra una entidad muy poco frecuente; se cuenta con muy poca información acerca de la asociación de la isquemia mesentérica como hallazgo de presentación del SAFC, y en revisiones sistemáticas en PubMed y Medline, no se encontraron artículos que hablaran de la 


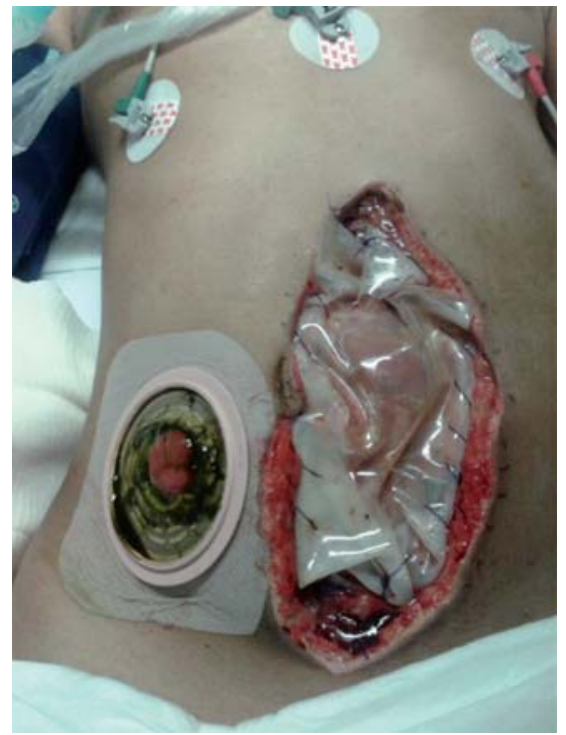

Figura 3. Bolsa de Bogotá e ileostomía terminal.

asociación del SAFC y el consumo de cocaína; además, no existen datos del porcentaje de pacientes con SAFC que comiencen con isquemia mesentérica aguda, siendo relevante mencionarlo, ya que así ocurrió en nuestro caso.

En pacientes jóvenes, en los diagnósticos diferenciales de un cuadro de abdomen agudo que comience súbitamente con deterioro rápido del nivel de conciencia y del estado hemodinámico, muy pocas veces se llega a pensar en isquemia mesentérica aguda como causa del cuadro, y menos una enfermedad reumatológica como factor primario. En este momento, los estudios de imagen en un paciente inestable se quedan obsoletos, ya que el tiempo de espera adquiere una vital importancia, aunque hay que recordar que el estudio diagnóstico de mayor sensibilidad y especificidad para la isquemia mesentérica aguda es la angiografía mesentérica, con $90 \%$ y hasta $98 \%$, respectivamente, según las publicaciones consultadas. ${ }^{10}$

Nosotros realizamos ileostomía terminal, abdomen abierto con colocación de bolsa de Bogotá y no entero-entero anastomosis aunque la cavidad abdominal se encontraba limpia; pensamos que en este caso fue la mejor decisión, ya que en ese momento no se contaba con la causa primaria del cuadro; esto deja como experiencia que en diagnósticos donde al cirujano le queda la duda de si la isquemia

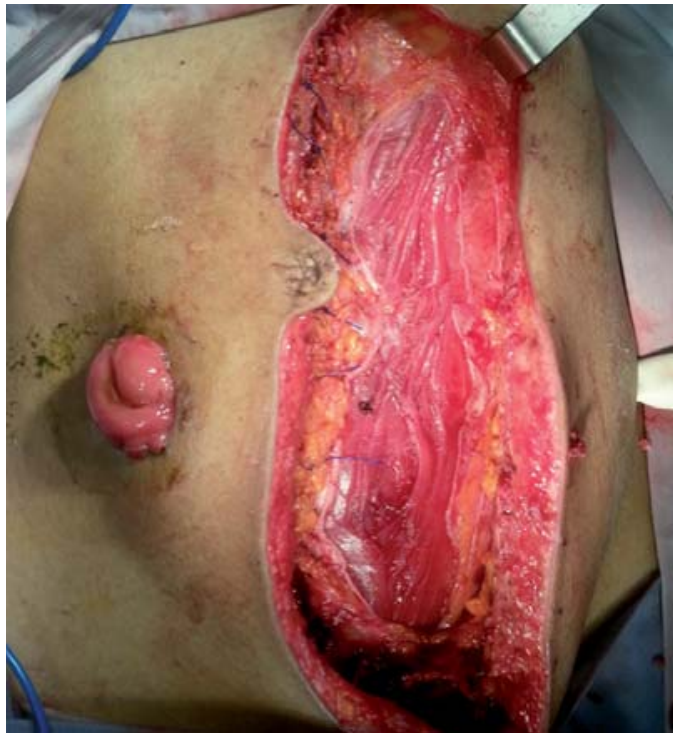

Figura 4. Cierre de pared mediante técnica de Gibson.

continuará o no conoce la causa primaria de la misma, siempre se debe decidir por realizar un "second look", por la posibilidad de neoisquemia y una nueva resección intestinal.

\section{CONCLUSIÓN}

La isquemia mesentérica aguda secundaria al SAFC representa una entidad sumamente rara con mortalidad elevada y, por lo tanto, se requiere de un tratamiento quirúrgico oportuno y una alta presunción diagnóstica para mejorar la supervivencia; siempre se deberá de tener en cuenta en pacientes jóvenes con pocos o nulos factores de riesgo predisponentes.

\section{CONFLICTO DE INTERESES}

Los autores declaran no tener ningún conflicto de intereses.

\section{REFERENCIAS}

1. Dhir V, Pinto B. Antiphospholipid syndrome: a review. J Mahatma Gandhi Inst Med Sci. 2014; 19: 19-27.

2. Nayer A, Ortega LM. Catastrophic antiphospholipid syndrome: a clinical review. J Nephropathol. 2014; 3: 9-17.

3. Giannakopoulos B, Krilis SA. The pathogenesis of the antiphospholipid syndrome. N Engl J Med. 2013; 368: 1033-1044. 
4. Cervera R, Espinosa G, Cordero A, Oltra MR, Unzurrunzaga $A$, Rossiñol $T$, et al. Intestinal involvement secondary to the antiphospholipid syndrome (APS): clinical and immunologic characteristics of 97 patients: comparison of classic and catastrophic APS. Semin Arthritis Rheum. 2007; 36: 287-296.

5. Uthman I, Khamashta M. The abdominal manifestations of the antiphospholipid syndrome. Rheumatology. 2007; 46: 1641-1647.

6. Atanassova PA. Antiphospholipid syndrome and vascular ischemic (occlusive) disease: an overview. Yonsei Med J. 2007; 48: 901-926.

7. Cojocaru M, Cojocaru IM, Silosi I, Vrabie CD. Gastrointestinal manifestations in systemic autoimmune disease. Maedica (Buchar). 2011; 6: 45-51.

8. Amigo-Castañeda MC. Tratamiento del síndrome antifosfolipídico, ¿a quién, cuándo y cuánto tiempo? Reumatol Clin. 2005; 1 Supl 2: S52-S58.
9. Carbajal-Rodríguez L, Vargas-Quevedo E, RamírezMayans JA, et al. Síndrome de Budd-Chiari (SBC) y su asociación con el síndrome antifosfolípidos (SAF). Acta Pediatr Mex. 2005; 26: 302-307.

10. Sánchez-Fernández P, Mier-Díaz J, Blanco-Benavides R. Isquemia mesentérica aguda. Semblanza de una enfermedad agresiva. Rev Gastroenterol Mex. 2000; 65: 26-28.

\author{
Correspondencia: \\ Dr. Eduardo Cordero Estrada \\ Salvador Quevedo y Zubieta Núm. 750, \\ Col. La Perla, 44620, Guadalajara, \\ Jalisco, México. \\ Tel: 0453314111287 \\ E-mail: eduardocordero10@hotmail.com
}

